Diabetologe 2011 · 7:606-606

DOI 10.1007/s11428-011-0826-5

Online publiziert: 3. Dezember 2011

(c) Springer-Verlag 2011

\section{B. Nowotny}

Institut für Klinische Diabetologie, Deutsches Diabetes-Zentrum an der

Heinrich-Heine-Universität, Leibniz-Zentrum für Diabetesforschung, Düsseldorf

\title{
Zusammenhang von ektoper Lipidspeicherung und $\beta$-Zell-Funktion
}

\section{Originalpublikation}

Van der Zijl NJ, Goossens GH, Moors CC et al (2011) Ectopic fat storage in the pancreas, liver, and abdominal fat depots: impact on $\beta$-cell function in individuals with impaired glucose metabolism. J Clin Endocrinol Metab 96:459-467

Dass eine ektope Lipidspeicherung in Organen, insbesondere gezeigt in Leber, Muskel und Herz, zu funktioneller Beeinträchtigung wie Insulinresistenz und diastolischer Dysfunktion führen kann, ist bekannt. Mechanistisch geht man im Moment davon aus, dass diese Einlagerung durch ein Überangebot an freien Fettsäuren („free fatty acids“, FFA) zustande kommt, die vollständig oxidiert werden können und bei gleichzeitigem Bestehen einer Speicherstörung im Fettgewebe in anderen Geweben abgelagert werden. $\mathrm{Ob}$ eine solche Lipidablagerung im Pankreas ebenso auftritt und auch zu funktionellen Beeinträchtigungen führt, ist im Moment noch unklar. Bisher gibt es nur einige wenige Publikationen, die teilweise einen inversen Zusammenhang zwischen Parametern der $\beta$-Zell-Funktion im oralen Glukosetoleranztest (oGTT) und Lipidspeicherung in Gesunden sowie in Personen mit gestörtem Glukosemetabolismus, aber nicht bei Typ-2-Diabetes, belegen. In der vorgestellten Studie wurde nun versucht, $\beta$-Zell-Funktion, Insulinsensitivität und Pankreasfett mit den jeweiligen Goldstandardmethoden von Personen mit gestörtem Glukosemetabolismus und gesunden Kontrollen zu erfassen.

\section{Studiendesign und Ergebnisse}

Es wurden 19 Personen mit kombinierter Glukosestoffwechselstörung („,impaired fasting glucose/impaired glucose tolerance“, IFG/IGT), 29 mit isolierter IFG und 16 alters-, geschlechts- und Body-MassIndex (BMI) angepasste Kontrollpersonen ohne Stoffwechselstörung (normale Glukosetoleranz, NGT) im oGTT untersucht. Die Insulinsensitivität wurde mit einem hyperinsulinämischen, euglykämischen "clamp“ bestimmt, die $\beta$-ZellFunktion durch einen hyperglykämischen Clamp, aus dem die „first phase insulin secretion " und die "second phase insulin secretion“ berechnet wurden. Des Weiteren wurde ein Argininstimulationstest zur Erfassung der maximalen sekretorischen Kapazität durchgeführt. Der Fettgehalt in Pankreas und Leber wurde mit ${ }^{1} \mathrm{H}-$ Magnetresonanzspektroskopie ( ${ }^{1} \mathrm{H}-\mathrm{MRS}$ ) mithilfe eines 1,5-T-MRT der Fa. Siemens unter Verwendung entsprechender Spulen bestimmt. Dabei fand sich ein Pankreasfettgehalt von im Mittel 7,6\% in der NGT-Gruppe gegenüber 12,1\% (IFG) und 22,4\% (IGT). Auch der Leberfettgehalt stieg über die 3 Gruppen stetig an, während der Anteil an subkutanem und viszeralem Fett identisch war. Während die Insulinsensitivität, gemessen als M-Wert im Clamp, signifikant positiv mit Pankreasfett, Leberfett ebenso wie viszeralem und subkutanem Fett korrelierte, fand sich keine Korrelation zwischen den Parametern der $\beta$-Zell-Funktion und einem der Fettdepots. Obwohl sich initial eine Korrelation zwischen dem Dispositionsindex $(\beta$-Zell-Funktion korrigiert um die bestehende Insulinsensitivität) zeigte, verschwand diese nach Adjustierung auf Alter und BMI.

\section{Kommentar}

Diese Studie konnte unter Verwendung von Goldstandardtechniken zur Erfassung von Insulinsensitivität und -sekretion ihre früher auf Basis von oGTT-abgeleiteten Parametern gefundene Assoziation von Pankreasfett und gestörter $\beta$-ZellFunktion nicht bestätigen. Obwohl die Ergebnisse der Funktionsuntersuchungen den Erwartungen aus publizierten Daten entsprechen und auch der Pankreasfettanteil erhöht ist, scheint dies zunächst nicht in einem direkten Zusammenhang zu stehen. Ob dies wirklich der Fall ist oder es hier aber an den technischen Voraussetzungen scheitert, bleibt offen. Auch wenn die ${ }^{1} \mathrm{H}-\mathrm{MRS}$ gegen den Goldstandard der Anfärbung validiert wurde und der parenchymale Triglyzeridgehalt zumindest in der Maus gut mit dem Fettgehalt in den Langerhans-Inseln selbst korrelliert, kann nicht zwischen Fett in den Inseln oder im exokrinen Parenchym unterschieden werden. Neben diesen gegebenen technischen Problemen bleibt auf der anderen Seite natürlich die Frage offen, ob - und wenn ja in welcher Konstellation - die in vitro und in vivo gefundenen Effekte von FFA auf die $\beta$-Zell-Funktion mit einer ektopen Lipidspeicherung im Zusammenhang stehen.

\section{Korrespondenzadresse \\ Dr. B. Nowotny}

Institut für Klinische Diabetologie, Deutsches Diabetes-Zentrum an der Heinrich-Heine-Universität, Leibniz-Zentrum für Diabetesforschung Auf'm Hennekamp 65, 40225 Düsseldorf bettina.nowotny@ddz.uni-duesseldorf.de

Interessenkonflikt. Die korrespondierende Autorin weist auf folgende Beziehung hin: Reisekostenübernahme von sanofi-aventis. 